\title{
Impulsive Control of Tumor Growth via Nonlinear Model Predictive Control Using Direct Multiple Shooting
}

\author{
Bence Géza Czakó and Dániel András Drexler and Levente Kovács
}

\begin{abstract}
Nonlinear Model Predictive Control (NMPC) is utilized to compute optimal administration protocols for chemotherapeutic treatment. By using model-based methods, the side effects of the drug can be mitigated in conjunction with a decrease in treatment expenses. The designed controller was able to provide the protocol in the form of impulses that can model administration by injection, using a smooth approximation of the Dirac delta distribution. For the implementation of the NMPC algorithm, Direct Multiple Shooting (DMS) was chosen so that the computational time of the problem remains reasonable. Numerical effects on the stability of the computation were discussed, with a solution for each issue present. The controller was also tested on virtual patients, with data from mice experiments, which concluded in applicable treatment protocols.
\end{abstract}

\section{INTRODUCTION}

Physiological control is a flourishing area of control engineering. The field includes topics such as diabetes control [1], automated anesthesia [2] or optimal control of tumor growth. Cancerous diseases entail high mortality rates worldwide including the European Union with a predicted 1.410.000 deceases in 2019, according to [3]. While the tendency shows a fall in mortality, which could be partially attributed to improved medicine, the vast majority of people are still treated with conventional methods until this day, which has adverse side effects. These side effects can be mitigated by optimizing the therapy which produces individual administration protocols. By minimizing the amount of drug used during the treatment, expenses can be reduced as well which can alleviate the financial burden on healthcare. The optimization can be tackled by the use of optimal control methods, which can calculate the dosage protocols while retaining the effects of disturbances on the treatment.

There are several examples concerning optimal tumor growth regulation using chemotherapy [4] or angiogenic inhibition [5]. Solutions in the literature vary from classical linear techniques [6] to sophisticated modern control methods, such as TP-LPV control [7], $H_{\infty}$ control [8] or feedback linearization [9]. However, a controller can not be designed without a proper mathematical description of the problem. Recently, a minimal model of tumor growth under chemotherapeutic treatment was developed by Drexler et al. where the parameters were estimated from mice experiments [10]-[12]. The main motivation of the developed model was to have a simple model that can describe the dynamics,

The Authors are with the Physiological Controls Research Center within the Research, Innovation and Service Center of Obuda University, Budapest, Hungary czako.benceephd.uni-obuda.hu drexler.danielenik.uni-obuda.hu

kovacseuni-obuda.hu with a relatively small number of parameters to facilitate identifiability and practical application of the model. While the model covers the most significant aspects of tumor growth on a macroscopic level, it does not encapsulate the effect of drug resistance. Hence, in order to obtain stable results, we assume that no resistance occurs during the treatment.

The choice of the model was motivated by its simplicity and the availability of realistic model parameters, based on mice experiments. There are numerous tumor growth models in the literature - for some recent developments see e.g., [13]-[15]. Nevertheless, these usually lack parameter values validated with real measurements and the equations are much more complex, which is not beneficial for neither identification nor controller design.

Our previous works concerned designing optimal dosage protocols for angiogenic inhibition, for which an NMPC was utilized, using the Hahnfeldt model [16]. Since the two problems share similar traits, NMPC was employed for chemotherapy optimization as well. One apparent issue was that the trajectory generation of the NMPC was very slow due to its implementation. Another issue arose from the continuous nature of the controller. Traditionally, NMPC uses piecewise-constant input signals as optimization variables. Nonetheless, a more realistic approach in therapy optimization would be the use of impulse signals that model the effect of injection treatment as opposed to a continuous type. Only a few examples exist for such a solution in optimal cancer treatment literature, using impulsive control based methods in angiogenic therapy [17], chemotherapy [18], or immunotherapy [19].

In Section II, the minimal model is presented, which is followed by an introduction of the NMPC technique, in conjunction with the impulsive action of the controller in Section III. The operation of the controller is described in Section IV, where different numerical aspects of the problem are discussed in conjunction with results on the virtual patients and the computational time of the optimization.

\section{MINIMAL MODEL OF TUMOR GROWTH}

The model that describes the tumor dynamics was taken from [10]. The states under consideration are the living tumor cells, the dead tumor cells, and the drug level. Model equations contain the effect of living tumor proliferation, tumor cell necrosis, dead cell washout, the effect of the drug with saturation (pharmacodynamics) and drug depletion, using mixed-order pharmacokinetics (also called capacitylimited pharmacokinetics) [20]. Mixed-order pharmacokinet- 
TABLE I: Model parameters and their dimensions

\begin{tabular}{ccc}
\hline Parameter & Unit & Description \\
\hline$a$ & $1 /$ day & Tumor growth rate \\
$n$ & $1 /$ day & Tumor necrosis rate \\
$b$ & $1 /$ day & Drug efficacy rate \\
$E D_{50}$ & $\mathrm{mg} / \mathrm{kg}$ & Effective median dosage \\
$w$ & $1 /$ day & Dead tumor cell washout \\
$c$ & $1 /$ day & Clearance rate of the drug \\
$K_{B}$ & $\mathrm{mg} / \mathrm{kg}$ & Michaelis-Menten constant \\
$b_{\kappa}$ & $\mathrm{mg} /\left(\mathrm{kg} \cdot \mathrm{day} \cdot \mathrm{mm}^{3}\right)$ & Modified efficacy rate \\
\hline
\end{tabular}

ics expresses that for low drug concentrations, the dynamics is first-order, while for large concentrations, the dynamics is zero-order, which is true for the pharmacokinetics of many drugs [21, p. 132]. The equations defining the dynamics are

$$
\begin{aligned}
& \dot{x}_{1}=(a-n) x_{1}-b \frac{x_{1} x_{3}}{E D_{50}+x_{3}} \\
& \dot{x}_{2}=n x_{1}+b \frac{x_{1} x_{3}}{E D_{50}+x_{3}}-w x_{2} \\
& \dot{x}_{3}=-c \frac{x_{3}}{K_{B}+x_{3}}-b_{\kappa} \frac{x_{1} x_{3}}{E D_{50}+x_{3}}+u
\end{aligned}
$$

where $x_{1}$ is the living tumor volume in $\mathrm{mm}^{3}, x_{2}$ is the dead volume in $\mathrm{mm}^{3}, x_{3}$ is the level of drug in the host measured in $\mathrm{mg} / \mathrm{kg}$ and $u$ is the injection rate in $\mathrm{mg} /(\mathrm{kg}$. day). Description of the parameters with their corresponding dimensions can be seen on Table I and explanation for the terms in the differential equations can be found in [10].

Model parameters were estimated from animal experiment data, where breast cancer was targeted with pegylated liposomal doxorubicin (PLD) in mice [22]. The estimation was performed using the Stochastic Approximation ExpectationMaximization (SAEM) algorithm and the concrete results can be found in [10], [11]. The algorithm maximizes the likelihood of each parameter using all the experimental data during the optimization iteratively. As such, each set of model parameters can be considered as a realization of a random variable from the underlying distribution that was obtained from the SAEM method.

\section{Nonlinear Model Predictive CONTROL}

Nonlinear Model Predictive Control is an optimal control methodology that can be utilized to compute optimal administration protocols for chemotherapeutic treatment. NMPC has a finite prediction horizon that can predict the evolution of the system by utilizing the mathematical model of the process [23]. This prediction is used in a predefined cost function in order to calculate an optimal sequence of control signals that are able to steer the system into the desired region. The sequence is calculated by minimizing the cost function using an optimization algorithm, which allows the use of constraints both on the inputs and states. Nonlinearity means that the algorithm supports the use of nonlinear models and constraints, as opposed to its linear counterpart. In practice, this leads to the use of more complicated numerical techniques, because no analytical solution can be carried out in the vast majority of cases. The cost function and constraints also imply the use of nonlinear optimization algorithms, such as the interior-point algorithm or Sequential Quadratic Programming (SQP). There are many ways to decrease the computational time of the calculations, although the Direct Multiple Shooting (DMS) method, in particular, is the state of the art technique, which provides a remedy to the computational burden.

\section{A. Direct Multiple Shooting}

The DMS is a continuous formulation of the NMPC framework, which is a simultaneous approach meaning that the optimization and the integration of the model are done in parallel [24]. First, the controls are discretized on a finite interval and assumed to be constant. By the use of these controls and artificial initial values, $N$ ODEs are solved which can be interpreted as trajectory pieces in the finite horizon. The continuity of these pieces is ensured by $N+1$ constraints in the nonlinear program which leads to a valid trajectory of the system at a feasible solution. The discretized control signal can be written as:

$$
u(t)=q_{i}, t \in\left[t_{i}, t_{i+1}\right]
$$

with the associated ODEs:

$$
\begin{aligned}
\dot{x}_{i}(t) & =f\left(x_{i}(t), q_{i}\right), t \in\left[t_{i}, t_{i+1}\right] \\
x_{i}\left(t_{i}\right) & =s_{i}
\end{aligned}
$$

where $x_{i}$ denotes the states of the system on the $i$ th time interval and $s_{i}$ is the artificial initial value of the $i$ th segment. The cost function can be defined as a functional of a stage cost on each prediction intervals:

$$
L_{i}\left(s_{i}, q_{i}\right)=\int_{t_{i}}^{t_{i+1}} \ell\left(x_{i}\left(t_{i} ; s_{i}, q_{i}\right), q_{i}\right) \mathrm{d} t
$$

with stage cost:

$$
\ell\left(x_{i}, q_{i}\right)=\lambda y_{i}+\xi q_{i}
$$

where $y_{i}$ is the total volume of the tumor $\left(y=x_{1}+x_{2}\right)$ on the $i$ th time segment and $\lambda, \xi>0$ are the weighting scalars. Continuity of the pieces can be ensured by imposing the conditions $s_{i+1}=x_{i}\left(t_{i+1} ; s_{i}, q_{i}\right)$ as a constraint for the optimization. The optimal control problem can be summarized as follows:

$$
\begin{aligned}
& \min _{s, q} \quad J_{N}(s, q):=\sum_{n=0}^{N-1} \ell\left(x_{i}, q_{i}\right)+E\left(s_{N}\right) \\
& \text { s.t. } \\
& s_{0}-x_{0}=0, \\
& s_{i+1}-x_{i}\left(t_{i+1} ; s_{i}, q_{i}\right)=0 i=0, \ldots, N-1, \\
& h_{i}\left(s_{i}, q_{i}\right) \geq 0 i=0, \ldots, N-1, \\
& r\left(s_{N}\right)=0,
\end{aligned}
$$

where $E\left(s_{N}\right)$ denotes the terminal weight, which is zero in this case. The first equality constraint represents the measurement, which is followed by the continuity constraints. The third and fourth constraints are the path and 
terminal constraints respectively, from which input (path) constraints were considered in order to avoid overdosing the patient. In accordance to the literature, the upper bound $q_{\max }$ must be smaller than $8 \mathrm{mg} / \mathrm{kg}$, which is the maximum tolerable dosage of PLD, and usually specified as $q_{\max } \in$ $\{2,4,6\}$ [25]. Lower bound must also be included in the optimization, because one can not extract drug from the patient, hence the interval can be given as $0 \leq q_{i} \leq 2$ for each control input in the sequence, which are the path constraints $h_{i}\left(s_{i}, q_{i}\right)$. Combining variables $s_{i}$-s and $q_{i}$-s into a single vector $w=\left[s_{1}, \ldots, s_{N}, q_{1}, \ldots, q_{N-1}\right]$, and using the notation $g_{i}\left(s_{i+1}, s_{i}, q_{i}\right):=s_{i+1}-x_{i}\left(t_{i+1 ; s_{i}, q_{i}}\right)$, one can rearrange the constraints in the problem as $g(w)=$ $\left[g_{0}, \ldots g_{i}, \ldots g_{N}, g_{N+1}\right]$ with $g_{0}$ being the initial value embedding $\left(s_{0}-x_{0}=0\right)$ and $g_{N+1}=r\left(s_{N}\right)=0$. While the bound constraints must be also included in the path constraint vector theoretically, most optimization algorithms can handle it as an additional input argument in practice. By these modifications, the problem can be reformulated as a classical nonlinear program:

$$
\begin{array}{cl}
\min _{w} & J_{N}(w) \\
\text { s.t. } & g(w)=0, \\
& 0 \leq q_{i} \leq 2
\end{array}
$$

This nonlinear program can be solved by nonlinear constrained optimization algorithms. One can also support the optimization algorithm with gradient matrices of the objective function and constraints, which has a block sparse structure that leads to faster and more reliable computations. Nevertheless, in many cases, the use of numerical differentiation can be fast and accurate, while the symbolical computations of these gradient matrices can be difficult without the use of a computer algebra system.

\section{B. Impulsive action}

The NMPC scheme discussed previously will provide piecewise-constant input signals for the rate of administration. In the case of chemotherapeutic treatment, this will be insufficient, since in practice infusion based administration is omnipresent. This entails that a different definition of the input signal must be introduced, which can model such an aspect of the therapy. One must be aware that in NMPC the constant nature of input signals is just a matter of choice and can be arbitrarily replaced by other piecewise continuous signals [23]. The idea for the impulsive control is to represent the Dirac delta distribution with a continuous approximation that can be used to model the infusion based administration. The main reason for the approximation is that numerical solvers can not perform well with discontinuities present in the system, hence the following bump function based estimate was utilized:

$$
u= \begin{cases}\frac{\zeta}{2 \varepsilon}\left(1+\cos \left(\frac{\pi(t-\xi)}{\varepsilon}\right)\right), & t_{0} \leq t \leq t_{0}+2 \varepsilon \\ 0, & \text { elsewhere }\end{cases}
$$

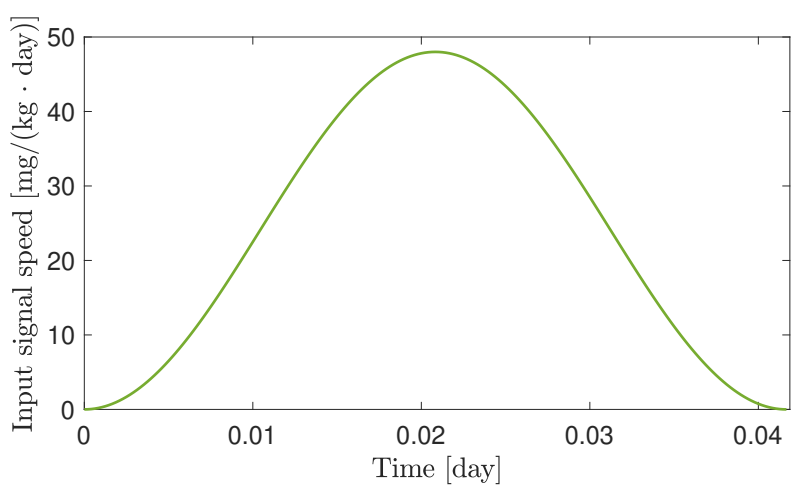

Fig. 1: The unitary impulse function

where $\varepsilon$ controls the approximation, $\xi=t_{0}+\varepsilon$ is the shifting term, $\zeta$ is responsible for the scaling and $t_{0}$ is the starting time of the impulse. Such a bump function is compactly supported and continuous so that the function vanishes at the endpoints (as opposed to Gauss distribution for example). The area of the curve is unitary, meaning that the integral of the impulse equal to $1 \mathrm{mg} / \mathrm{kg}$ PLD dosage. The particular approximation can be seen on Fig. 1, where $\zeta=1, \varepsilon=$ $(1 / 24) / 2$ and $t_{0}=0$. The value of $\varepsilon$ is chosen according to the duration of a bolus administration which is usually between $30-90$ minutes, hence in this particular case an hour-long treatment was considered. It can be seen in Figure 1. that the function is shifted so that it always lies at the beginning of each optimization interval. As it was indicated in [26], the times of injections are determined by the doctors, so that it can not be a free optimization variable. Instead of that, $\zeta$ is used for the optimization, which allows one to increase the area of the function thus injecting more drug into the patient. This leads to $q_{i}=\zeta_{i}$ in the optimization process which computes the scaling terms $\zeta_{i}$ for each $\left[t_{i}, t_{i+1}\right]$.

\section{Simulation}

Simulations were conducted using MATLAB and the optimization was performed with fmincon. The model was integrated using ode45 with a 100 equidistant integration point on a 1 day interval, which gives the piecewise solution to equation (3). From the definition of the cost function (5), it is clear that the controller aims to drive the volume as close to $0 \mathrm{~mm}^{3}$ as possible while minimizing the effect of the input. In the case of NMPC, tuning the design parameters of the controller is usually problem specific and hence done experimentally. The prediction horizon was set to $N=4$ which proved to be sufficient. It must be mentioned that in theory, increasing the horizon enhances the stability of the system, however, by practice, it was not the case because the algorithm was prone to get stuck in local minima frequently. The issue can be tackled by restarting the algorithm from a different initial point $w_{0}$ or adjusting the integration tolerances in the optimization. Therefore, if the algorithm halts in a local minimum, a different initial value, $w_{0}^{\text {new }}$, is chosen randomly from a normal distribution with $w_{0}^{\text {new }} \sim$ $\mathcal{N}\left(w_{0}, 0.5 \sqrt{w_{0}}\right)$ for a given number of iteration $n_{\max } \leq 10$ 
until a feasible minimizer is obtained. In the cases where the stochastic approach fails to provide meaningful results, the integration tolerances can be fine-tuned which solves the problem.

For the stage cost, parameters were also determined experimentally, from which $\lambda=1$ and $\xi=0.1$. This particular choice will result in the first control signal to be large in amplitude, compared to the consecutive actions. This effect can be mitigated by decreasing the value of $\lambda$ while ensuring that the system retains its stability. In particular, $\lambda \approx 0.3$ provided stable results with no windup in the control signal at the beginning for each virtual PLD specimen.

One advantage of the design is that the length of the optimization intervals $\Delta t=t_{i+1}-t_{i}$ can be modified arbitrarily. By doing so, daily, weekly or different administration protocols can be obtained by the algorithm. It must be noted, however, that longer intervals can increase the error of integration in the prediction, which can cause singularities in the solution. This can be alleviated by fine-tuning the error tolerances of the integration as well. Besides the problem of the error accumulation, lower bounds on the inputs $0 \leq$ $\zeta_{i}$ can pose another issue. During the optimization, the algorithm might choose some $\zeta_{i}$ to be zero. Because the unforced response of the system grows exponentially, this can cause singularities during iterations corresponding to longer time intervals. In order to overcome this issue, a different lower bound can be set, so that the system remains stable during each iteration. For the weekly administration, $0.05 \leq \zeta_{i}$ provided convergent results for each virtual patient PLD. Examples for the weekly administration can be seen in Figure 2, simulated for $7 \cdot 30=210$ days from PLD2 to PLD5. On the corresponding administration protocols, a spike represents the integral of one impulse on a control interval that is the total amount of drug injected for a single infusion. One can see that in some cases, for example, the input signals are not computed correctly for PLD3. This can be attributed to the aforementioned numerical errors in the objective function. Nevertheless, the solutions are feasible, which implies the stability of the closed-loop response [23]. In the examples PLD2 and PLD5, the simulation time was not enough for the system to reach steady-state, however, they will decay eventually.

Drug resistant virtual patients were also examined, which can be seen in Figure 3. Prediction interval was set to $\Delta t=1$ day because larger horizons lead to non-convergent behavior of the algorithm. While the growth of PLD8 can be brought to a stable equilibrium point, PLD1 grows exponentially which can not be altered by the controller significantly. In the case of PLD9, linear growth can be achieved instead of exponential behavior.

There was a major decrease in terms of computational time as well. For the above mentioned parameter choices, the algorithm was able to calculate the optimization task at each control cycle in $\sim 1$ second on average, which was performed by an Intel Core i7-8550U, $1.8 \mathrm{GHz}$ processor. In Table II, one can see the increase of computational time for longer prediction horizons in the case of PLD2.

\begin{tabular}{cc}
\hline $\mathrm{N}$ & $\begin{array}{c}\text { Mean computational time } \\
(\mathrm{sec})\end{array}$ \\
\hline 7 & 1.0247 \\
5 & 0.5671 \\
3 & 0.2047 \\
2 & 0.1129 \\
\hline
\end{tabular}

TABLE II: Mean computational time of one control cycle for PLD2

Results can be compared with previous works, concerning antiangiogenic treatment using NMPC with single shooting, since the two models share similar traits [27]. In that case, the computational time of the optimal solution usually took 1 day to compute, for $N=10$, which was inefficient, compared to the DMS implementation. The additional decrease in computational time can be achieved, if one considers the result $w$ of the optimization to be the initial guess $w_{0}$ for the next iteration, which is usually called a warm start in the MPC literature.

In conclusion, the proposed control method is able to compute optimal dosage protocols in each case. Results, however, are affected by numerical imprecisions, especially for longer prediction horizons or larger initial tumor volumes, which can be solved by the aforementioned changes in the tolerance of the computations. Nevertheless, a more robust algorithm would be favorable, which can be achieved by non-dimensionalizing the model equations.

\section{CONCLUSion}

An NMPC controller was developed using impulsive input actions that can produce optimal administration protocols for chemotherapy. The impulsive action of the controller was introduced by using a continuous approximation of the Dirac delta distribution. The design was tested on the minimal model, for each virtual patient, identified from mice experiments. Results showed that the controller was able to produce an optimal input signal sequence for each PLD patient, even in the case of resistant behavior. The effect of numerical inaccuracies was discussed with solutions proposed for each problem arisen.

In future works, the stability of the computations should be improved in general, by either non-dimensionalizing the model, or introducing stabilizing constraints in the optimization routine. It must also be mentioned, that for the NMPC, the whole state must be measured, which is not possible directly from the given model. Because the model is observable, a nonlinear observed can be designed so that the whole state can be reconstructed from the measurements.

\section{ACKNOWLEDGMENT}

The authors would like to express their thanks to the Membrane Protein Research Group of the Hungarian Academy of Sciences for providing the measurement data. This project has received funding from the European Research Council (ERC) under the European Union's Horizon 2020 research and innovation programme (grant agreement No 679681). 

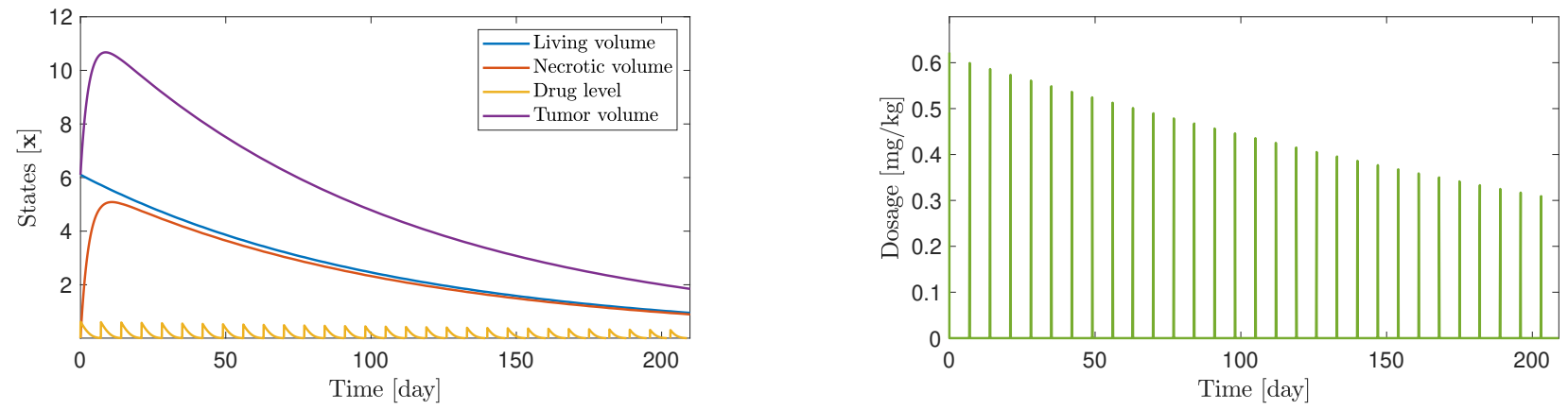

(a) PLD2
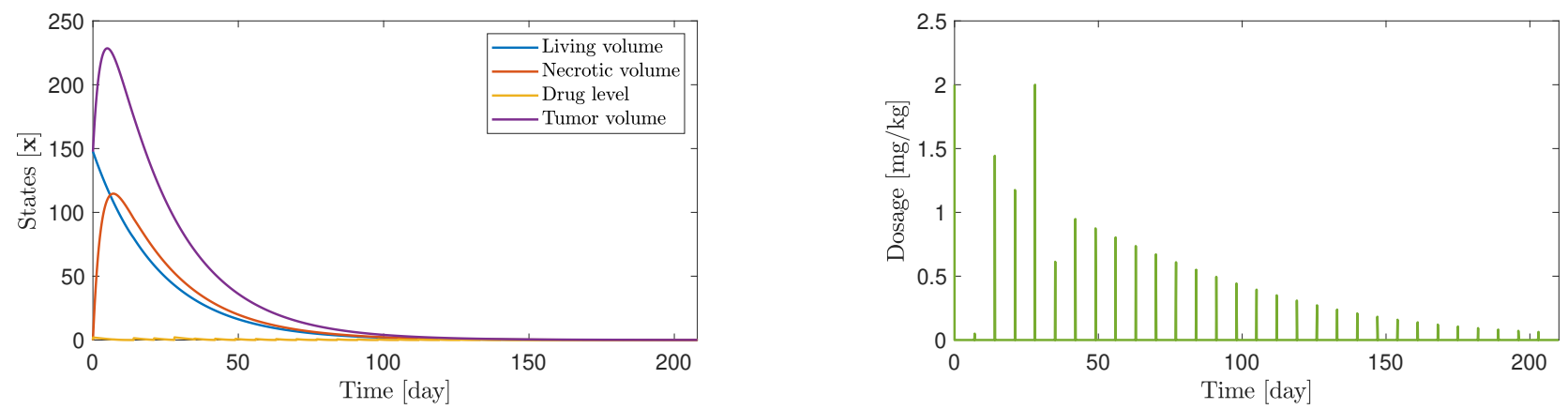

(b) PLD3
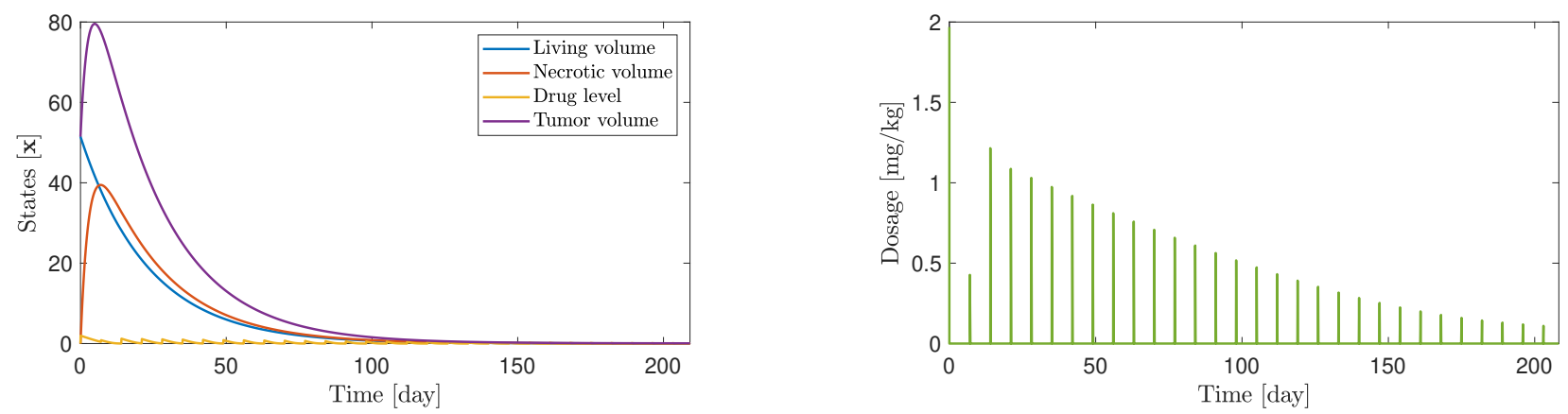

(c) PLD4
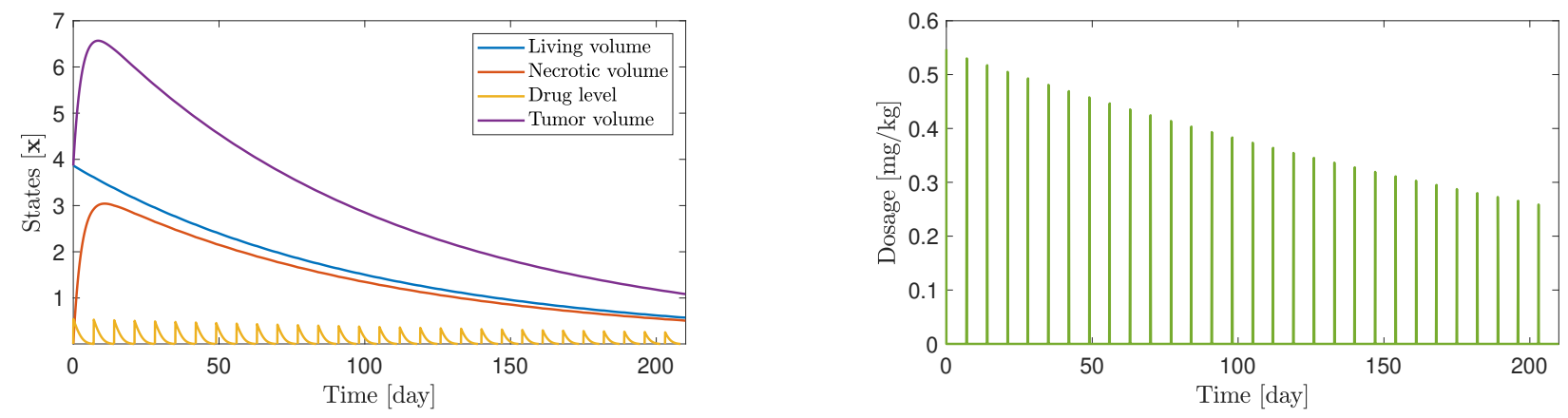

(d) PLD5

Fig. 2: Results of the optimization for PLD2-PLD5. The evolution of the states $x_{1}, x_{2}, x_{2}$ in conjunction with the total volume of the tumor $y=x_{1}+x_{2}$ (left) and the individual dosages presented as the integral of the calculated inputs $u$, given in $[\mathrm{mg} / \mathrm{kg}]$ (right). Design parameters were: $N=3, \lambda=0.7, \xi=0.1, \Delta t=7$. 


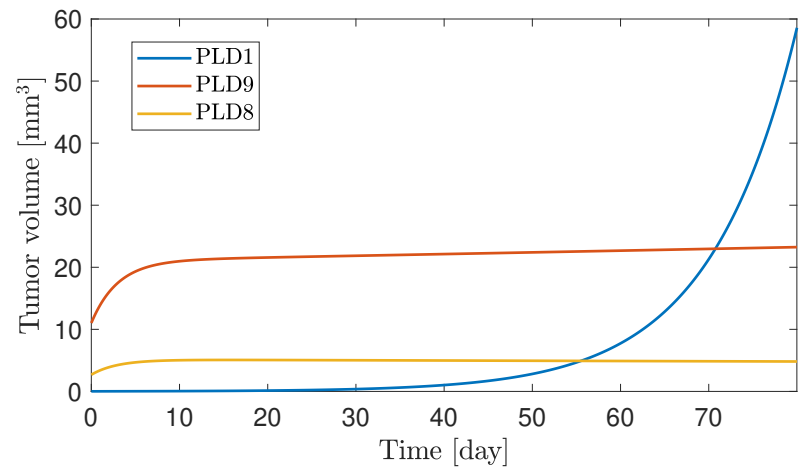

Fig. 3: Operation of the controller on drug resistant specimen, using $\Delta t=1$ day. Design parameters were: $N=3, \lambda=$ $0.7, \xi=0.1, \Delta t=7$.

Bence G. Czakó was supported by the UNKP-18-3/II. New National Excellence Program of the Ministry of Human Capacities.

\section{REFERENCES}

[1] E. Dassau, H. Zisser, R. A. Harvey, M. W. Percival, B. Grosman, W. Bevier, E. Atlas, S. Miller, R. Nimri, L. Jovanovic, and F. J. Doyle, "Clinical evaluation of a personalized artificial pancreas," Diabetes Care, vol. 36, no. 4, pp. 801-809, nov 2012. [Online]. Available: https://doi.org/10.2337/dc12-0948

[2] T. M. Hemmerling, "Automated anesthesia," Current Opinion in Anaesthesiology, vol. 22, no. 6, pp. 757-763, dec 2009. [Online]. Available: https://doi.org/10.1097/aco.0b013e328332c9b4

[3] M. Malvezzi, G. Carioli, P. Bertuccio, P. Boffetta, F. Levi, C. L. Vecchia, and E. Negri, "European cancer mortality predictions for the year 2019 with focus on breast cancer," Annals of Oncology, mar 2019. [Online]. Available: https://doi.org/10.1093/annonc/mdz051

[4] G. W. Swan, "Role of optimal control theory in cancer chemotherapy," Mathematical Biosciences, vol. 101, no. 2, pp. 237-284, oct 1990. [Online]. Available: https://doi.org/10.1016/0025-5564(90)90021-p

[5] N. Nath, T. Burg, D. M. Dawson, and E. Iyasere, "Optimizing antiangiogenic therapy for tumor minimization," in Proceedings of the 2010 American Control Conference. IEEE, jun 2010. [Online]. Available: https://doi.org/10.1109/acc.2010.5531040

[6] L. Kovacs, P. Szalay, T. Ferenci, J. Sapi, P. Sas, D. Drexler, I. Harmati, B. Benyo, and A. Kovacs, "Model-based control algorithms for optimal therapy of high-impact public health diseases," in 2012 IEEE 16th International Conference on Intelligent Engineering Systems (INES). IEEE, jun 2012. [Online]. Available: https://doi.org/10.1109/ines.2012.6249892

[7] G. Eigner, M. Siket, A. Szakal, I. Rudas, and L. Kovacs, "Discrete LPV modeling of diabetes mellitus for control purposes," in 2018 IEEE International Conference on Systems, Man, and Cybernetics (SMC). IEEE, oct 2018. [Online]. Available: https://doi.org/10.1109/smc.2018.00438

[8] D. A. Drexler, J. Sápi, and L. Kovács, "H $\infty$ control of nonlinear systems with positive input with application to antiangiogenic therapy," IFAC-PapersOnLine, vol. 51, no. 25, pp. 146-151, 2018. [Online]. Available: https://doi.org/10.1016/j.ifacol.2018.11.096

[9] _ - "Positive nonlinear control of tumor growth using angiogenic inhibition," IFAC-PapersOnLine, vol. 50, no. 1, pp. $15068-15073$, jul 2017.

[10] D. A. Drexler, T. Ferenci, A. Lovrics, and L. Kovács, "Tumor dynamics modeling based on formal reaction kinetics," Acta Polytechnica Hungarica, vol. 16, pp. 31-44, 2019.

[11] D. A. Drexler, T. Ferenci, A. Lovrics, and L. Kovács, "Comparison of Michaelis-Menten kinetics modeling alternatives in cancer chemotherapy modeling," IEEE 13th International Symposium on Applied Computational Intelligence and Informatics (SACI2019), vol. , no. -, pp. $000027-000032$, May 2019.
[12] D. A. Drexler, J. Sápi, and L. Kovács, "Modeling of tumor growth incorporating the effects of necrosis and the effect of bevacizumab," Complexity, pp. 1-11, 2017.

[13] R. Aogo and F. Nyabadza, "Modelling the dynamics of HIVrelated non-Hodgkin lymphomas in the presence of HIV treatment and chemotherapy," Mathematical Methods in the Applied Sciences, vol. 41, no. 18, pp. 8385-8406, 2018.

[14] K. Chakravarty and D. C. Dalal, "Stability analysis of drug dynamics model: A mathematical approach," International Journal of Biomathematics, vol. 12, no. 4, p. 1950043, May 2019.

[15] J. Paez Chavez, B. Gurbuz, and C. M. A. Pinto, "The effect of aggressive chemotherapy in a model for HIV/AIDS-cancer dynamics," Communications in Nonlinear Science and Numerical Simulation, vol. 75, pp. 109-120, 2019.

[16] B. Czakó and L. Kovács, "Nonlinear model predictive control using robust fixed point transformation-based phenomena for controlling tumor growth," Machines, vol. 6, no. 4, p. 49, oct 2018. [Online] Available: https://doi.org/10.3390/machines6040049

[17] D. A. Drexler, J. Sápi, and L. Kovács, "Optimal discrete time control of antiangiogenic tumor therapy," IFAC-PapersOnLine, vol. 50, no. 1, pp. 13504-13 509, Jul. 2017. [Online]. Available: https://doi.org/10.1016/j.ifacol.2017.08.2337

[18] H.-P. Ren, Y. Yang, M. S. Baptista, and C. Grebogi, "Tumour chemotherapy strategy based on impulse control theory," Philosophical Transactions of the Royal Society A: Mathematical, Physical and Engineering Sciences, vol. 375, no. 2088, p. 20160221, Mar. 2017. [Online]. Available: https://doi.org/10.1098/rsta.2016.0221

[19] A. Cappuccio, F. Castiglione, and B. Piccoli, "Determination of the optimal therapeutic protocols in cancer immunotherapy," Mathematical Biosciences, vol. 209, no. 1, pp. 1-13, Sep. 2007. [Online]. Available: https://doi.org/10.1016/j.mbs.2007.02.009

[20] W. J. Jusko, "Pharmacokinetics of capacity-limited systems." Journal of Clinical Pharmacology, vol. 29, pp. 488-493, 1989.

[21] J. T. DiPiro, W. J. Spruill, W. A. Wade, R. A. Blouin, and J. M. Pruemer, Concepts in Clinical Pharmacology, 5th ed. American Society of Health-System Pharmacists, 2010.

[22] A. Füredi, K. Szebényi, S. Tóth, M. Cserepes, L. Hámori, V. Nagy, E. Karai, P. Vajdovich, T. Imre, P. Szabó, D. Szüts, J. Tóvári, and G. Szakács, "Pegylated liposomal formulation of doxorubicin overcomes drug resistance in a genetically engineered mouse model of breast cancer," Journal of Controlled Release, vol. 261, pp. 287-296, Sep. 2017. [Online]. Available: https://doi.org/10.1016/j.jconrel.2017.07.010

[23] L. Grüne and J. Pannek, Nonlinear Model Predictive Control. Springer International Publishing, 2017. [Online]. Available: https://doi.org/10.1007/978-3-319-46024-6

[24] M. Diehl, H. G. Bock, H. Diedam, and P.-B. Wieber, "Fast Direct Multiple Shooting Algorithms for Optimal Robot Control," in Fast Motions in Biomechanics and Robotics, Heidelberg, Germany, 2005. [Online]. Available: https://hal.inria.fr/inria-00390435

[25] W. J. Aston, D. E. Hope, A. K. Nowak, B. W. Robinson, R. A. Lake, and W. J. Lesterhuis, "A systematic investigation of the maximum tolerated dose of cytotoxic chemotherapy with and without supportive care in mice," BMC Cancer, vol. 17, no. 1, Oct. 2017. [Online]. Available: https://doi.org/10.1186/s12885-017-3677-7

[26] F. Cacace, V. Cusimano, and P. Palumbo, "Optimal impulsive control with application to antiangiogenic tumor therapy," IEEE Transactions on Control Systems Technology, pp. 1-12, 2018

[27] B. Czako, J. Sápi, and L. Kovács, "Model-based optimal control method for cancer treatment using model predictive control and robust fixed point method," in 2017 IEEE 21st International Conference on Intelligent Engineering Systems (INES), Oct 2017. 